\title{
When Does Invasion Mean the War is Lost?
}

\author{
Claudius Conrad, MD, $\mathrm{PhD}^{1}$ and Jean-Nicolas Vauthey, MD, FACS ${ }^{2}$ \\ ${ }^{1}$ Division of Surgical Oncology, Department of Surgery, Massachusetts General Hospital, Harvard Medical School, \\ Boston, MA; ${ }^{2}$ Division of Surgery, Department of Surgical Oncology, The University of Texas MD Anderson Cancer \\ Center, Houston, TX
}

The significance of macrovascular invasion (MVI) in hepatocellular carcinoma (HCC) is ill defined. Although the Barcelona Clinic Liver Cancer Classification System classifies disease associated with MVI as stage $\mathrm{C}$ and recommends that patients with MVI be offered palliative therapy with the oral multikinase inhibitor sorafenib, several centers have reported successful liver resection with good outcomes in patients with MVI. ${ }^{1}$ The paper by Roayaie et al. ${ }^{2}$ provides an excellent opportunity to revisit the significance of MVI in HCC. We congratulate the authors for their thorough evaluation of their results, critical discussion of their data, and comprehensive summary and analysis of the available relevant literature.

The authors performed a retrospective analysis of 165 patients with Child's class A cirrhosis and MVI treated with primary hepatic resection during the period from 1992 to 2010. Median survival time after surgery was 13.1 months, and the 5-year survival rate was $14 \%$. These outcomes compare favorably to the 8-month median survival of patients with MVI who received sorafenib during the important SHARP trial (108 patients, $36 \%$ of the patients in the sorafenib group). ${ }^{3}$ In their multivariate analysis, Roayaie et al. ${ }^{2}$ determined that alfa-fetoprotein level greater than $30 \mathrm{ng} / \mathrm{mL}$, tumor size greater than $7 \mathrm{~cm}$, and more extensive vascular invasion were independent predictors of worse overall survival. Furthermore, hepatic vein or inferior vena cava invasion was associated with significantly worse overall survival following resection

This is an editorial to the article available at doi:10.1245/s10434013-3074-7.

(C) Society of Surgical Oncology 2013

Published Online: 10 July 2013

J.-N. Vauthey, MD, FACS

e-mail: jvauthey@mdanderson.org (median 4.7 months) than was portal venous invasion (median 9.2 months). Possible reasons for the poor outcomes of patients with hepatic vein or inferior vena cava invasion undergoing resection might be ready access of the tumor to the systemic venous circulation or the high morbidity of the surgery itself.

A question that could have been addressed in the paper in more detail is whether there are shades of gray when it comes to portal venous invasion by HCC. We think the answer is "yes." Ikai et al. ${ }^{4}$ published a paper in 2003 that showed that there are survival differences according to the order of portal branch involved, a parameter that allows accurate prognostication (Fig. 1). The Liver Cancer Study Group of Japan has adopted and incorporated this concept. Therefore, MVI of the portal vein is helpful for determining outcome, as shown by Roayaie et al., ${ }^{2}$ but might be defined more precisely as a discrete variable (tumor thrombus in first-, second-, or third-order branch) than as a qualitative variable (presence or absence of MVI).

An issue raised by the authors is the $60 \%$ rate of detection of MVI on preoperative imaging. Although the long time interval covered by the study (1992-2010) saw advances in operative techniques (the reported mortality rate of $28 \%$ for patients with hepatic vein or inferior vena cava involvement might be lower today) and the 2005 approval of sorafenib by the Food and Drug Administration, the preoperative detection sensitivity and specificity of Vp1-4 MVI might still only be around 70 and $80 \%$ respectively. ${ }^{5}$ Preoperative detection of major vascular invasion $(\mathrm{Vp} 3)$ is important to determine the need for preoperative transarterial chemoembolization (TACE). Roayaie et al. ${ }^{2}$ were surprised by the results of the study by Minagawa et al. ${ }^{6}$, in which 18 patients undergoing TACE plus liver resection were compared to 27 patients who did not undergo hepatectomy. This study was conducted at a center with specialized expertise in a cohort of patients 


\section{Tumor Thrombi in Portal Vein Branches}

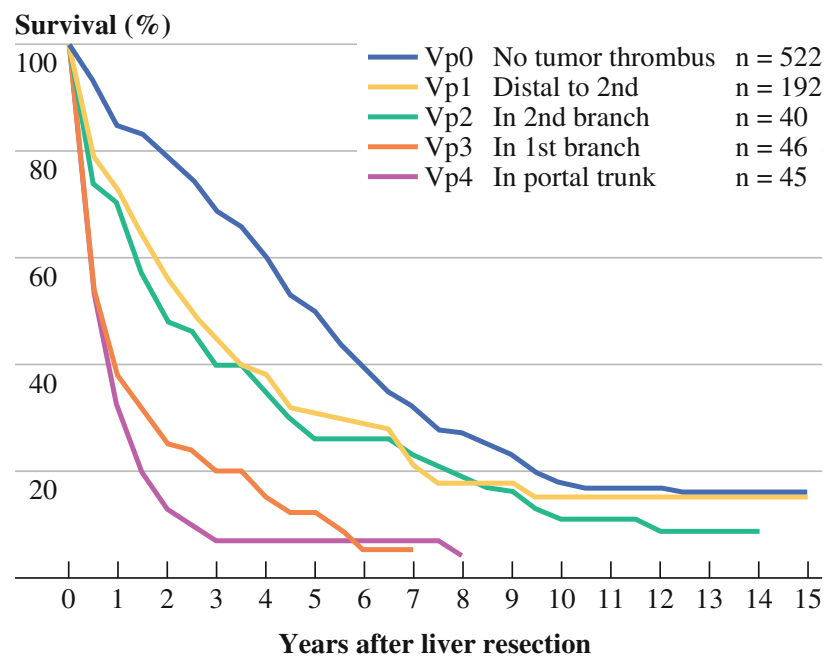

FIG. 1 Survival of patients with portal vein invasion depends on which order of portal vein branch is involved (adapted from Ikai et al. ${ }^{4}$ )

with advanced first-order portal branch involvement (Vp3), and the 5-year survival rate after hepatectomy was $42 \%$. In our experience with patients with major vascular TACE can solidify the tumor thrombus and this may prevent tumor dissemination in the FLR and reduce early intrahepatic recurrence. Several studies from Asia have been published on the strategy of TACE followed by liver resection. Although the degree of vascular invasion often not detailed in the publication, some reports describe the use of TACE to convert HCC from unresectable to resectable. ${ }^{7,8}$ This strategy should be considered in experienced centers.

Some of the parameters that might be useful for patient stratification are available in the pathology report, and information about these factors, had it been reported by Roayaie et al. ${ }^{2}$, would have been of interest. These include details about the underlying fibrosis and extent of vascular invasion in the portal veins (Vp1-4) or the hepatic veins (Vv1-3), ${ }^{9}$ which have been shown to be important predictors of outcome. ${ }^{10,11}$ Currently, presence or absence and extent of vascular invasion and degree of fibrosis are the two main predictors of outcome of HCC. Large solitary tumors without vascular invasion are classified as UICC/ AJCC stage 1, whereas tumors with vascular invasion single or multiple are classified as stage 2. Multiple tumors greater than $5 \mathrm{~cm}$ or tumors with $\mathrm{Vp} 3$ are classified as stage 3 . These criteria best stratify HCC in the east and west as universal predictors of a single disease entity after resection and transplantation. ${ }^{10,12}$ When looking beyond histopathologic parameters as we enter the arena of molecular and genomic medicine, we will have to find accurate molecular markers of outcome that will likewise stratify HCC prognosis. For example, recent work identified paraoxonase 1 as a serum biomarker for microvascular invasion in $\mathrm{HCC}$ using quantitative proteomic analysis. ${ }^{13}$

Preclinical studies suggest that novel prognostic markers and putative therapeutic targets can be found in the family of cell cycle regulators, including key compounds of iNOS cross-talk with IKK/NF- $\kappa \mathrm{B}$ and RAS/ERK pathways (also targeted by sorafenib), the ERK inhibitor DUSP1, FOXM1 with its targets, and ubiquitin ligases. ${ }^{14}$ Another promising marker, osteopontin, is an integrin-binding glycophosphoprotein that is expressed in transformed malignant epithelial cells and is believed to be involved in many physiologic cellular functions, such as regulation of migration, invasion, and metastasis of tumor cells as well as their survival. Elevated expression of osteopontin at the transcript (mRNA) level has been reported to be associated with the prognosis of patients with HCC. ${ }^{15}$ Although these approaches are promising, further research in this field is necessary.

Roayaie et al. ${ }^{2}$ provide an excellent paper that revisits many important issues pertaining to HCC prognostication and stratification of patients for surgical therapy. Our outcomes for patients with HCC involving the hepatic veins at the level of the confluence or the inferior vena cava have been poor, similar to what the authors report, and therefore we recommend resection only in selected cases (Vp1-3 with good performance status and early or no cirrhosis). Preoperative TACE should be considered in patients with $\geq \mathrm{Vp} 3$. Although the authors identify tumor size, alfa-fetoprotein level, and extent of vascular invasion as predictors for worse prognosis, in our view, they do not preclude selected patients from resection and might provide outcomes superior to best medical therapy.

\section{REFERENCES}

1. Torzilli G, Belghiti J, Kokudo N, et al. A snapshot of the effective indications and results of surgery for hepatocellular carcinoma in tertiary referral centers: is it adherent to the EASL/AASLD recommendations? An observational study of the HCC East-West study group. Ann Surg. 2013;257:929-37.

2. Roayaie S, Jibara G, Taouli B, Schwartz ME. Resection of hepatocellular carcinoma with macroscopic vascular invasion. Ann Surg Oncol. doi:10.1245/s10434-013-3074-7.

3. Llovet JM, Ricci S, Mazzaferro V, et al. Sorafenib in advanced hepatocellular carcinoma. $N$ Engl J Med. 2008;359:378-90.

4. Ikai I, Yamamoto Y, Yamamoto N, et al. Results of hepatic resection for hepatocellular carcinoma invading major portal and/ or hepatic veins. Surg Oncol Clin N Am. 2003;12:65-75, ix.

5. Sorrentino P, Tarantino L, D'Angelo S, et al. Validation of an extension of the international non-invasive criteria for the diagnosis of hepatocellular carcinoma to the characterization of macroscopic portal vein thrombosis. J Gastroenterol Hepatol. 2011;26:669-77.

6. Minagawa M, Makuuchi M, Takayama T, et al. Selection criteria for hepatectomy in patients with hepatocellular carcinoma and portal vein tumor thrombus. Ann Surg. 2001;233:379-84. 
7. Choi SB, Kim KS, Park YN, et al. The efficacy of hepatic resection after neoadjuvant transarterial chemoembolization (TACE) and radiation therapy in hepatocellular carcinoma greater than $5 \mathrm{~cm}$ in size. J Korean Med Sci. 2009;24:242-7.

8. Kishi Y, Saiura A, Yamamoto J, et al. Preoperative transarterial chemoembolization for hepatocellular carcinoma. Hepatogastroenterology. 2012;59:2295-9.

9. Liver Cancer Study Group of Japan. General rules for the clinical and pathological study of primary liver cancer. 2nd ed. Tokyo: Kanehara \& Co, Ltd; 2003. p. 1-77.

10. Vauthey JN, Lauwers GY, Esnaola NF, et al. Simplified staging for hepatocellular carcinoma. J Clin Oncol. 2002;20:1527-36.

11. Pawlik TM, Poon RT, Abdalla EK, et al. Hepatectomy for hepatocellular carcinoma with major portal or hepatic vein invasion: results of a multicenter study. Surgery. 2005;137:403-10.
12. Vauthey JN, Ribero D, Abdalla EK, et al. Outcomes of liver transplantation in 490 patients with hepatocellular carcinoma: validation of a uniform staging after surgical treatment. J Am Coll Surg. 2007;204:1016-27.

13. Huang $\mathrm{C}$, Wang Y, Liu S, et al. Quantitative proteomic analysis identified paraoxonase 1 as a novel serum biomarker for microvascular invasion in hepatocellular carcinoma. J Proteome Res. 2013;12:1838-46.

14. Frau M, Biasi F, Feo F, Pascale RM. Prognostic markers and putative therapeutic targets for hepatocellular carcinoma. Mol Aspects Med. 2010;31:179-93.

15. Pan HW, Ou YH, Peng SY, et al. Overexpression of osteopontin is associated with intrahepatic metastasis, early recurrence, and poorer prognosis of surgically resected hepatocellular carcinoma. Cancer. 2003;98:119-27. 